Voix et Images

volxetimages

\title{
Saint-Denys Garneau : La parole et le poète
}

\section{Georges Riser}

Volume 5, numéro 2, hiver 1980

Yves Thériault

URI : https://id.erudit.org/iderudit/200207ar

DOI : https://doi.org/10.7202/200207ar

Aller au sommaire du numéro

Éditeur(s)

Les Presses de l'Université du Québec

ISSN

0318-9201 (imprimé)

1705-933X (numérique)

Découvrir la revue

Citer cet article

Riser, G. (1980). Saint-Denys Garneau : La parole et le poète. Voix et Images, 5(2), 291-306. https://doi.org/10.7202/200207ar d'utilisation que vous pouvez consulter en ligne.

https://apropos.erudit.org/fr/usagers/politique-dutilisation/ 


\section{Saint-Denys Garneau: La parole et le poète}

Largement développé plus tard, le thème de la parole est assez neuf en poésie, vers les annèes 1935-37. Saint-Denys Garneau aborde ce thème diune manière très individuelle, comme son problème personnel; dans ses poèmes, dans son Journal, il n'en disserte pas en philosophe, encore moins en linguiste, il parle simplement d'une sorte d'expérience dans laquelle il est impliqué. Cette parole est toute tournée vers le poète, comme le poète est tourné vers elle, pour la mieux connaître, pour se connaitre et se dire èn elle.

L'étude de quelques poèmes permettra de saisir cette relation délicate entre le poète et la parole poètique. Ces textes datent de l'automne 1935 et n'ont pas été retenus pour le recueil Regards et jeux dans l'espace ${ }^{1}$. Ce sont Silence (O.PR, 156), d'octobre, et Te voilà verbe (O.PR, 158) de décembre, à quoi il faut ajouter l'essai en prose, Monologue fantaisiste sur le mol (O.PR, 289), à un an de distance, soit fin 1936. Leur contenu, comparé à celui d'autres couvres de la même époque, révèle que Saint-Denys Garneau ría pas, sur la question, une pensée très claire, cohèrente el bien formulèe. II procède par tâtonnements et adopte même des positions contradictoires. Rien d'élonnant chez lui, qu'on sait habité par l'instabilité et les contradictions; rien d'étonnant non plus en raison même du sujet: c'est en usant d'une parole poétique qu'il analyse la forme de celte parole et sa relation avec elle. La parole humaine, d'autre part, comporte un certain nombre de relations: celle des mots entre eux, celle des mots avec les choses auxquelles ils réfèrent, celle des mots avec l'homme parlant, poète ou non, et celle du locuteur (ou de l'écrivain) avec son destinataire. Les poèmes étudiés ici ne traitent explicitement que de la relation de la parole avec le poète et, par endroits, de celle des mots avec les choses. D'autres poèmes, non retenus ici, posent le problème du message communiqué et mal reçu.

Saint-Denys Garneau a passé l'été 1935 à crayonner avec grâce et bonheur ses "Esquisses en plein air" et des poèmes qui n'y ont pas trouvé 
place. En octobre, Le jeu (O.RJE, 10) et Nous ne sommes pas des complables (O.RJE, 11) manifestent l'enthousiasme du jeune poète, visionnaire comme l'enfant et comme lul créateur, puisant dans "sa boite à jouets pleine de mots"... Octobre, c'est surtout Riviere de mes yeux (O.RJE, 13) et les trois vers de Baigneuse (O.PR, 161). El tout à coup, Silence (O.PR, 156):

Toutes paroles me deviennent intérieures

Et ma bouche $[\ldots]$

$[\ldots]$ ne prononce plus ces paroles dans le temps, des paroles en passage,

Mais se ferme et garde comme un trésor

ses paroles

[...]

Ses paroles qui ne sont pas du temps

Si Saint-Denys Garneau dit ici "ma bouche", il ne dit pas "mes paroles", mais "toutes" paroles, "ces» paroles, "ses" paroles. II n'emploie du reste jamais cette expression “ mes paroles", dans aucun de ses poèmes, ni dans son Journal. II traite toujours de ces questions avec un certain détachement, à la $3^{e}$ personne, comme si l'homme, en lui, jugeait l'artiste ou l'écrivain. Ces paroles apparaissent donc posées comme une réalité indépendante de son étre. On peut en déduire que le poète perçoit une certaine distance entre ses paroles el lui. Certes, elles ne sont pas encore extériorisées, prononcées dans la chaine sonore; il les garde " intérieures". II désigne ainsi ce discours, cette parole muette, inarticulée, qui se déroule en nous pour donner corps à nos concepts et aux images qui surgissent en nous. Plus que tout autre homme, le poète est le lieu où s'essaye cette parole hésitante, inaudible tant qu'elle n'a pas reçu sa forme articulèe dans la chaine sonore et surtout dans l'écriture. Mais dèjà, avant son existence dans le temps, cette parole nomme les choses et entre avec elles en un rapport étroit de signification.

Saint-Denys Garneau avoue d'emblèe que uloutes paroles (lui) deviennent intérieures". Ce "deviennent w exprime une mutation en train de s'opérer, et dans le sens inverse de l'ordre normal de la parole. Au lieu de passer de l'état intérieur à sa forme extérieure, la parole intériorise ici son jaillissement spontané et enferme "dans le coffre" ce qui, de soi, est destiné à la naissance dans le dire poétique. La parole fonde bien l'être des choses, selon le mot de Heidegger, mais c'est pour les cacher aux regards, pour les enfourr dans le ccur du poète ou elles vont vivre de leur vie secrète. Tout le poème développe cette décision de ne rien communiquer, de ne rien faire exister désormais en dehors du poète. Le premier vers, dejà très explicite, est repris par les vers suivants:

[...] ma bouche se ferme comme un coffre

[...] se ferme et garde comme un Irésor ses paroles.

et par les derniers vers, où la forme du verbe indique le fait accompli:

Ma bouche est fermée comme un coffre

Sur les choses que mon âme garde intimes

Qu'elle garde incommunicables

Et possède ailleurs 
"Incommunicables", " coffre", "bouche fermée", voilà clairement dit que ce que le poète porte en lui et qui a déjà revêtu la forme floue el incertaine de la "parole intérieure". tout cela va rester "intérieur" et ne naîtra jamais à la lumière du discours poètique explicite. La distance constatée plus haut entre le poète et "ces" paroles est corrigèe, tempérée par cette intériorisation nuancée de possession.

Le poète choisit l'intériorisation à cause des effets destructeurs du temps passager et "salissant". Pour lui, seules les choses "hors l'atteinte du temps" passager confinent à l'absolu, dans la purelé imputrescible de l'éternité. Les "paroles dans le temps" seront toujours "des paroles en passage", mouvantes, fragiles, participant à la précarité des choses qui naissent et qui meurent. Saint-Denys Garneau semble oublier ainsi, pour les besoins de sa cause, que les paroles prononcées dans le temps ont reçu une existence. mème fugitive, ont pu être entendues et reçues; il oublie que les paroles poétiques sont destinées à revevoir dans le poème écrit une forme stable, propre à défier les siècles, tandis que les paroles intérieures ont une existence des plus précaires! L'option philosophique sous-jacente est encore plus visible dans l'accusation portée contre "le temps salissant " qui entraine toutes choses dans son impureté. L'ordonnance inévitable à la mort des choses et des êtres surtout est plus qu'une faiblesse, elle est une souillure, la marque biblique du péché. Si donc le poète décide de ne plus parler, c'est pour sauvegarder les qualités du «trésor"

On voit ainsi apparaitre une relation étroite entre le trésor-parole ef le trésor-choses. Saint-Denys Garneau tente de définir ce trésor incommunicable par nature, et procède par approximations:

Ses paroles qui ne sont pas du temps

Mais qui représentent le temps dans l'èternel,

Des manières de représentants

Ailleurs de ce qui passe ici,

Des manières de symboles

Des manières d'évidence de l'èternité qui passe ici.

Des choses uniques, incommensurables,

Qui passent ici parmi nous mortels

Pour jamais plus jamais...

A trois reprises, "des manières de..." trahissent la difficultè de trouver l'expression adéquate. En effet, comment nommer dans le temps ces " paroles qui ne sont pas du temps"? C'est une gageure. Mais plus intèressante est la tentative d'exprimer le rapport de signification entre les mots qui nomment. qui informent pour nous une réalité, et cette réalité elle-même, au plan ontologique. Si les paroles "représentent", elles rendent présent, elles tiennent la place de quelque chose d'autre, de nature différente, et situé "ailleurs". Elles sont un signe. - ici "des manières de symboles", - qui réfèrent si ontologiquement a la réalite mystérieuse, qu'elles lui confèrent comme une "évidence". Ainsi, il suffit de tenir fermement toutes paroles intérieures pour tenir du même coup, avec la mème vérité, la mystérieuse 
réalıté des choses et de l'ailleurs. Le poème ètablıt un rapport très ètroit entre paroles et choses, puisque garder les paroles et garder les choses, c'est tout un.

Quelle est, d'autre part, celte précieuse et mystérieuse réalité ? Elle ne désigne pas clairement l'image des choses et des ètres formée en nous, ni leurs rapports mutuels, ni non plus des sentiments. Le point de vue est philosophique et existentiel à la fois. En effet, les paroles "représentent le temps dans l'èternel", elles sont "des manières d'évidence de l'éternité qui passe ici". Quatre fois revient l'expression "qui passe ici", reprenant celle "des paroles en passage" du troisieme vers. Elle est lièe à la notion du temps. à ce qui est mortel, à ce qui existe un jour pour disparaitre à jamais. Elle s'oppose immédiatement, à l'intérieur des mêmes vers. aux notions d'“éternel" et d'c ailleurs", qui s'épaulent l'une l'autre et se complètent. On pourrait se hasarder à donner la représentation suivante du róle des paroles:

les paroles

intérieures
CE QUI PASSE dans le TEMPS MORTELS

rendent présent L'ETERNEL
ICI

parmi nous

dans L'ETERNEL AILLEURS

Ce tableau met en lumière l'aspect anti-platonicien de la relation. Ce n'est pas l'Éternel, la parfaite réalité d'ailleurs, qui est représentè, rendu présent au sein du Temps, en subissant une sorte de réduction et d'affadissement; c'est le Temporel, tout ce qui passe ici et toul ce qui se passe parmi nous, qui est reprèsentè dans l'Eternel, dans l'Ailleurs, et qui en reçoit extension, accroissement jusqu'à l'infini et fixation hors des mutations dégradantes du temps.

La parole intérieure se révèle donc porteuse d'une réalitè précieuse en elle-mème, et fragile, * des choses uniques, incommunicables * et intimes ? qui seraient anéanties, ou du moins abîmées, si elles subissaient une inévitable réduction dans la forme d'une parole prononcée dans le temps. Le rapport a l'infini serait detruit, el il semble bien que ce soit cette dimension qui préoccupe d'abord Saint-Denys Garneau. Détruit aussi le rapport à l'esprit, si on donnait corps à la parole: ces choses "incommensurables" perdraient de leur extension et de leur plènitude. Thèse qui n'appartient pas en propre a notre poète: on y reconnait l'influence de Dom Bellot dont les thèses sont analysées dans L'Art spıritualiste (O.Pr. 240-243), el surtout celle de Jacques Maritain qui affirme, dans Frontières de la poésie, que "les choses sont mieux dans l'esprit qu'en elles-mêmes".

Le désir d'éterniser ce qui passe est assez commun à tout homme, à plus forte raison à tout poète. La référence à l'u Ailleurs" est plus rare et mérite attention. A deux reprises ${ }^{3}$ déjà. Saint-Denys Garneau a mentionné cette double aspiration à l'èternel et à l'ailleurs. Il est difficile d'admettre que cet Ailleurs puisse ici renvoyer à l'Autre Monde, l'éternel, celui de la croyance religieuse, où le péché et la mort n'ont justement plus de place. L'Ailleurs 
pourrait plutôt désigner ce monde précieux, idéal, perçu et capté par le poète "au-delà" de la réalité sensıble el comme le seul réel c. Ce pourrait être encore un autre moment de la vie du poète, où il sait avoir vécu une expérience extraordinaire, et qu'il cherche à retrouver, à faire revivre avec plus d'acuité qu'un pâle souvenir, à faire émerger des brumes du passé, comme dans les experiences de Baudelaire dans "La vie antérieure" et surtout celles de Proust. Pourquoi ne serait-ce pas enfin le lieu d'une a autre existence" du poète, quand se produit dans la conscience une sorte de dédoublement, comme Saint-Denys Garneau l'éprouvera lui-même de quelque façon, à en croire le Journal (O.J 408) en date du 15 novembre 1937 , et certains poèmes. tels Accompagnement (O.RJE. 34) ou Et maintenant (O.PR, 188). II est bien difficile de se prononcer, car toutes ces significations peuvent être vraies simultanément, tant est mystérieuse l'expérience du poète fondatrice de son œuvre.

II ne faut donc pas chercher ici une expression linguistique précise de la théorie du signe et du référent. Pour ce poète qui parle de son expérience, pour cet homme qui aime les choses et la possession des choses, les liens entre les choses et la parole apparaissent donc assez clairs. Les "choses" reçoivent déjà dans la parole intérieure une certaine existence, dans une relation si étroite que tenir les paroles cachèes, c'est cacher les choses elles-mêmes. De plus, celte parole confère aux choses périssables par nature une sorte de perfection et d'éternité particulièrement chère au poète. Mais les rapports se compliquent dès que le poète entre en jeu: les choses appartiennent au poète. les paroles ne paraissent pas siennes avec la même évidence; ce qui est trésor, ce sont les choses, non les paroles. Enfin, si tout le trésor est gardé intérieur, enfermé dans le coffre, cette intériorisation qui marque une relation spéciale avec le poète, est presque détruite par la diffèrence ontologique qui existe entre le contenu et le contenant, entre le poète et les paroles. On voit combien il est difficile, pour Saint-Denys Garneau, de cerner avec exactitude ce probleme complexe.

Les deux poèmes Parole sur ma lèvre (O.PR. 156) et Te voilà verbe (O.PR, 158) ont trop de points communs pour qu'on ne les étudie pas ensemble, même si le deuxième se déroule sur un mode plus agressif. Tous deux traitent de la parole prononcée, qui passe les lèvres. L'expression "toutes paroles", au pluriel, cède la place à la «parole" au singulier, comme si on passait du discontinu intérieur, non élaboré, à la chaine continue de la langue parlée. II sera mème question du "poème", pour souligner la forme plus organisée et radicalement extérieure; on rencontre enfin le terme "verbe", c'est-à-dire la parole entrevue dans son dynamisme et son efficacitè. Nous abordons ainsi une autre ètape de son existence et d'autres modes de sa relation au poete. 
Il est impossible de passer de Silence à ces deux poèmes sans avoir l'impression qu'il manque quelque chose, qu'on a saute un maillon dans l'enchainement de l'aventure. Maillon qui se rèvèle en creux par des allusions, des traces. Les deux poèmes revêtent la forme d'une apostrophe, d'une invective. d'une accusation méme. C'est dire qu'on s'adresse à une personne ou à quelque chose de personnifié. Les "paroles intérieures" ne portaient aucune trace de personnification, de relation vivante avec le poete. II faut croire qu'il s'en est instauré une, même si le poète n'en a pas parlé explicitement. D'autre part. le poète s'élève vivement contre la situation ectuelle, ce qui laisse supposer qu'il pouvait, qu'il devait en être autrement ¿ uparavant:

Parole sur la lévre déjá prends ton vol

[...] tu n'es plus à moi

Impuissant sur toi maintenant dès ta naissance Je me heurterai à toi maintenant Comme à toute chose etrangère.

Ces accusations portées contre la situation de amaintenant", - ce " maintenant " répétè parce qu'insupportable, - manifestent une rupture, une modification de la relation antérieure. Tout est changé depuis la " naissance " de la parole: maintenant elle n'est plus au poète - elle lui appartenait donc auparavant; maintenant il est impuissant vis-à-vis d'elle, - il avait donc sur elle puissance et autorite, il pouvait en disposer, comme dans $L \theta$ Jeu (O.RJE, 10) où il arrangeait les mots à sa fantaisie; maintenant il va se heurter à elle. alors qu'avant devaient régner accord et harmonie; elle sera pour lui comme une chose étrangère, alors qu'elle devait être la chose familière, fraternelle et intime; à son contact, il n'éprouvera plus le frisson fraternel, comme celui que lui procurait le contact de la femme aimée et bien connue, l'intimité est dissipèe après avoir existè. Le second poème souligne furtivement, mais en termes clairs, ce qu'on vient de déceler en creux, quand il fait du verbe une "projection" de son inconscient, et surtout « un fils", un " frère".

Une relation nouvelle se serait donc instaurée entre le poèle et sa parole poétique. Relation particulière, intime, fraternelle, presque filiale: n'est-elle pas comme un être qui nait en lui et de lui, au sens le plus fort du terme? Certes, la langue préexiste au locuteur, qui ne fait que puiser dans un réservoir très vaste, mais limıté. Or il advient cette chose prodigieuse: avec ces instruments de tout le monde, communs et uses, chacun invente, engendre sa propre parole, toujours neuve, aux formes et aux nuances infinies. Saint-Denys Garneau a donc raison de se crorre maitre de sa parole, à cause des rapports ètroits pressentis entre elle et lui. Les poèmes Le jeu et Tous ef chacun (O.PR. 157) parlent de cette activité créatrice du langage, le second poème laissant même entendre que tout n'est pas toujours réussite parfaite. Mais aujourd'hui, le poète est sensibilisè à autre chose: cette relation fraternelle naturelle est brisée et cède la place à une extériorité qui va devenir une veritable hostılite. 
La première accusation porte sur l'extériorité:

Parole sur ma lèvre déjá prends ton vol

Va-t'en extérieure...

tu ries plus à moi

Ainsi, dès son articulation informante, la parole devient "extérieure". C'est dans l'ordre des choses, el sans gravité au fond: hors du sein de sa mére, l'enfant reste son enfant. Mais ici te poète ressent cet ordre normal des choses comme une atteinte. Le ton est celui d'une invective, non d'une constatation. On dirait que le poète se fåche contre la parole quand, par sa nature méme et son dynamisme propre, elle vient à l'existence articulée ou écrite dans le poème, perdant ainsi les qualités lièes à son précédent état d'intériorité. Toutes les autres images employées, comme se heurter à une chose étrangère, expriment la mème attitude étrange et significative: SaintDenys Garneau ne reproche pas a la parole de trahir son contenu, mais d'être infidèle à lui, poète, sensible avant tout à la relation à la fois possessive et affective. L'exigence de l'adéquation parlaite, toujours insatisfaite, entre les moyens du langage et la réalité poétique des choses à exprimer, a hanté tous les poètes. Elle ne semble pas avoir effleuré Saint-Denys Garneau dans ces poèmes. Toutes les constatations d'échec qui émaillent poèmes, Journal, Correspondance, relèvent plutôt d'une incapacité radicale de son être, de ses propres difficultés; ou bien la faute en est aux autres. Jamais à la langue ellemême.

L'accusation s'aggrave: la parole n'est pas seulement extérieure ou étrangère, elle est "ennemie *:

Va-t'en extérieure, puisque tu l'es déjá

Parmi toutes ces portes fermées.

ennemie

[...]

Tu es déjá parmi l'inéluctable qui m'encercle

Un des barreaux pour mon etouffement.

Le " déjà " qui précède a ennemie ", et qu'on rencontre encore au premier vers el à l'avant-dernier, souligne a la fois la rapidité de la mutation, opérée au sein du mème vers, et la surprise devant la distance relationnelle et affective prise soudain par ce qui aurait dũ être si proche. Cette inimitié exprime non pas une qualité propre et caractéristique de la parole dite, mais la projection subjective de l'état d'âme du poète. Elle va se déployer en deux grands motifs, celui de l'encerclement et celui du monstre dévorant.

L'image de l'encerclement et de la fermeture renforce l'accusation d'extériorité, mais elle a sa signification propre. On s'attendrait à ce que le dire poétique soit ressenti comme une libération, un apaisement des tensions intérieures de la poésie qui "appelle au fond du cceur, pareille à une vie de surcroit rèclamant son droit à la parole dans la lumière", comme dit Anne Hébert dans "Poésie, solitude rompue". Or au lieu d'une ouverture, nous constatons une fermeture. Si la parole intérieure respectait les mouvances, la 
"forme changeante" de l'être du poète et des choses en lui, laarticulation dans la forme externe fixe toute signification, fige un seul de ses états d'âme fugitifs et transitoires, comme un instantané photographique, au détriment du mouvement qui respecterait mieux la vérité de l'être. Les portes se ferment, le poète est "encerclè". Bien plus, la parole se liguerait ainsi à tant d'autres éléments, en lui et autour de lui, pour former une prison de "barreaux" pour son étouffement. Enfin, l'image de l'encerclement envahit le deuxième poème, on la retrouve en ces vers:

\section{Et voilà le poème encore vide qui m'encercle \\ [...] \\ M'encercle d'une mortelle tentacule}

Ces accusations loutes subjectives, dont il serait intéressant de rechercher les motivations profondes, montrent que la relation personnelle et fraternelle est rompue au profit d'une douloureuse situation d'hostilité.

Si le poète se sent ainsi prisonnier de la parole prononcèe, pourquoi ne pas la reprendre et en dire une autre, autrement? Mais est-ce vraiment possible? L'artiste n'a pas entière autorité ni pleine décision dans le choix du mot $^{5}$, de la forme que revêt sa parole prononcée. et du sens ou de l'image qu'elle porte. Il y a en effet une part, toujours difficile à cerner, de jaillissement spontané et nécessaire du verbe qui échappe au contrôle conscient de l'artiste et qui constitue souvent sa vérité essentielle. D'autre part, comme le souligne Gaetan Picon, dans son Introduction à une esthétique de la littérature, la parole - ou l'écriture - modifie d'une certaine façon l'être de l'homme: une parole ne se retire jamais tout à fait, après avoir été dite; elle laisse une trace dans l'être profond de l'artiste. Saint-Denys Garneau avait donc raison de craındre la puissance et même la tyrannie de la parole prononcée.

La relation d'opposition se fait plus violente dans le poème To voilà verbe.

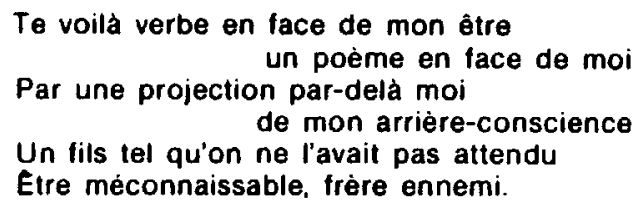

"En face" apparaît deux fois en ce premier vers. II révèle une position de face a face de deux êtres, dans une totalité évidente d'affrontement qui intensifie elle aussi l'image de l'encerclement et des portes fermées. Le "verbe " surgit "en face" de l'être du poète. Par le fait même, il est plus qu'une chose; il est doue, par référence à l'être du poète, d'une certaine qualité ontologique, dune certaine "densité substantielle" (0.J, 495), bref il est un autre Je en face du Je du poète. On sait, d'après le Journal et la Correspondance, le drame de Saint-Denys Garneau à cette époque quand il rencontrait d'autres êtres, des amis mème comme François Rinfret $(O . J .495,489)$ : celte rencontre se muait en une sorte d'affrontement entre la force rayonnante et insultante de l'être des autres et le vide. le néant de son âme détrulte. II esl certain qu'ici 
se produit le même transfert: le "verbe" en face de son ètre, c'est un autre Je immédiatement perçu en comparaison avec celui du poète; et par opposition à lui, il va se réveler dominateur, vorace, destructeur. On trouve donc ici une autre forme de personnification de la parole, mais les relations sont changejes.

Projeté hors des profondeurs du "centre" du poète, porteur de ce qui lui est le plus consubstantiel, le verbe n'en esl pas moins un "être méconnaissable "... Non pas en raison de sa forme nouvelle, extérieure, mass dans sa relation au poète. Car ce verbe "fils" ou "frère" est en réalité

Un fils tel qu'on ne l'avait pas attendu

[...] trère ennemi.

Deux images riches d'une opposition violente el tragique. Ce qui vient à l'existence dans le dire poétique n'est donc qu'un enfant non désirè, 6 l'enfant "agresseur" selon la terminologie courante. La symbiose naturelle du poète et de la parole. - de la mère et de l'enfant, - à la fois charnelle et spirituelle. est détruite au plan affectif, ce qui peut aller jusqu'à un rejet destructeur. La deuxième opposition est plus vigoureuse encore dans sa concision: "frère ennemi ". Saint-Denys Garneau reprend ici une image qui vient du fond des àges, des traditions mythologiques et bibliques. Elle a toujours exprimé la tragique et mortelle hostilite qui surgit au sein d'un groupe d'êtres fondés dans une unité de chair et de sang. puisée aux sources de la vie, et indestructible malgré toutes les haines.

Une dernière image paraît enfin, qui pousse à l'extrême ce drame du poète confronté au mot poètique. Telle une bète monstrueuse, telle une sorte de pieuvre géante, non nommèe mais suggérée par sa "mortelle tentacule". sa "bouche suçante", sa "ventouse ", le verbe ennemi encercle le poète d'un embrassement mortel, colle à sa chair, aspire son sang, le vide el l'anéantit.

Et voila le poème encore vide qui m'encercle

Dans l'avidite d'une terrible exıgence de vie.

M'encercle d'une mortelle tentacule,

Chaque mot une bouche suçante, une ventouse

Qui s'applique à moi

Pour se gonfler de mon sang

Peu importe la valeur et la justesse de cette image exacerbée et hallucinante pour caractériser la parole poètique. Le poème ne développe que l'aspect relationnel. Par deux fois.

Te voilà verbe en face de mon être

[...]

Et voilà le poème encore vide qui m'encercle

ces "voilà" manifestent eux aussi la douloureuse surprise du poète devant l'apparition de son bourreau, qu'il découvre trop tard pour s'en protéger. La "terrible exigence" traduit bien la panique qui s'empare du poète quand dans une même appréhension il saisit la puissance vorace et prımitıve du verbe et sa propre fabblesse, son rôle de victime. On devine enfin l'épuisement du 
poète subissant une douloureuse biophagie" qui le saigne, vivant et lucide. Le dernier vers,

\section{Et je nourrirai de moelle ces balancements}

dètachè du reste du poème, avec le verbe au lutur, dit bien la résignation de la victime et annonce peut-être la réalité future du poète "réduit à ses os" (O.PR, 173). Si tout ceci nous apprend peu de chose sur la parole poétique en elle-mème, c'est par contre très révélateur de l'état d'âme du poète en pleine crise spirituelle et morale.

Saint-Denys Garneau connait enfin un répit. Entre avril et août 1936, il est très occupé par des problèmes d'esthétique, en particulier celle de la peinture (O.J. 430-438). Cette longue réflexion a sans doute permis une maturation plus sereine des questions poétiques qui font l'objet de l'essai en prose, Monologue fantaisiste sur le mot (O. Pr, 289), ecrit probablement en novembre ou en décembre 1936 et paru dans le numéro de janvier-février 1937 de La Reléve. Cet essai méle beaucoup de choses: on y trouve reprises des expressions, des attitudes deja rencontrees dans les poèmes, et des allusions à l'expérience passée ; on y découvre des explicitations et, enfin, des considerations nouvelles. II y est question du "Mot", bien que la "Parole" apparaisse à la fin du texte, dans une certaine opposition avec ce terme. Le " mot " semble désigner ici la même réalité que les " paroles " de Silence, c'esta-dire les unités de langue les plus importantes, celles qui sont porteuses d'une signification essentielle. D'autre part, le Monologue ne fait pas de distinction entre la parole intérieure et celle qui franchit les lèvres. Cet essai, tout en caractérisant le "mot", développe surtout la diversité des relations entre le poète et le mot.

Saint-Denys Garneau refuse de voir dans le mot " une chose vide", ou un "simple instrument de désignation " qu'on découvrirait au terme d'une recherche. Pour lui, te mot est un être "vivant, substantiel", riche de mille rapports au monde. On n'a pas à le chercher: comme une personne, doué de spontanéité. il apparaît de lui-même, il lance des appels entendus par le poète. Cette "personne" est différenciée de celle du poète qui se réveille " en face du monde des mots" comme "en face d'un dieu qui sait ce que nous ne savons pas". On a donc bien affaire à un autre Je, de dimension plus grande que le poète, une sorte de divinité habitant une "région au-dessus". Le progrès est evident, par rapport aux poèmes où la personnification en un ètre vivant ne se devinait qu'à travers l'apostrophe et l'incarnation hostile en une sorte de vampire. Le climat est devenu serein, affectueux même, et la relation positive.

Chose ètrange, cet être se présente comme un être "vide». Le terme "vide", qui affectait déjà "le poème encore vide" de To voilà verbe, se 
retrouve ici dans le refus du mot comme "chose vide" et surtout dans les nombreuses allusions à la nécessité de le "remplir". Par trois fois justement. le Monologue rappelle "la terrible exigence" des mots. Exigence vitale, existentielle: le mot a besoin d'être nourri, il a grande "soif de substance", simplement pour exister et pour remplir sa fonction. Le poète avoue avoir appris à ses dépens - "il m'a fallu les combler, les nourrir de moi-mẽme" -. qu'il n'exprime rien avec les "inanimés-mots" du lexique, cimetière de mots exsangues. Existant pour dire l'homme et ses rapports au monde, le mot ne peut être vide de substance humaine; il n'est pas pure relation logique, pure forme sonore d'un concept, sans épaisseur ni densité historique. Comme dit Jean-Claude Renard. dans Notes sur la poesie, "un drame I'habite toujours". Plus celui “qui a empli le mot d'un contenu humain plus ou moins personnel el expérimentén est un homme de grande culture, humaniste ou poète, plus le mot acquiert de perfection et de grandeur.

Mais on ne nourrit pas le mot poétique de n'importe quelle substance anonyme. Ce n'est pas l'expérience des autres, la souffrance des autres, les questions des autres qui peuvent être une nourriture convenable. Pour SaintDenys Garneau comme pour tant d'autres poètes, it faut que cela vienne du poète lui-mème, que cela lui appartienne aussi essentiellement que son propre sang '. Méme si un poète ne peut jamais s'abstraire totalement de ce qu'il a assimilé de l'expérience des autres, c'est de son propre fond, en ce qu'il a d'original et d'absolument unique, qu'il doit combler le mot, pour que la langue, véhicule universel et commun à tous, devienne apprivoisée, individualisée et propre a fonder dans f'existence "les réseaux des fils invisibles dont le poète est le lieu". Ainsi, sans que l'image mythique ait été explicitée, on retrouve ici. plus affirmée et nettement pacifique, la relation de filiation déjà découverte. Le poète et la parole sont bien deux êtres distincts, différents, mais l'un vit de l'autre, est "nourri w de la substance de l'autre - et non dévoré - comme un enfant dans le sein de sa mère. Symbiose harmonieuse de deux ètres qui se complètent dans leur distinction, car la parole ne serait rien sans le sang du poète, et le poète ne serait pas poète sans celte parole née de lui et en lui.

Saint-Denys Garneau nous entraine encore plus loin par le biais de la connaissance. Sans employer les termes mémes de Claudel dans l'Art poétique, le poète montre que la théorie de la "co-naissance" lui est familière. Poète et parole poétique naissent ensemble à l'existence, ce qui leur confère une certaine connaturalité. Non seulement le poète connaît le mot, mais il le "reconnait". Cette reconnaissance affirme la préexistence de liens, "une certaine fraternité, communication vivante, une correspondance "... qu'on saisit immediatement dans la rencontre. Quel chemin parcouru depuis le jour où le verbe n'était qu'un "être méconnaissable" ! L'image des fées du conte, qui inaugure le Monologue, vient renforcer cette relation de connivence. Le conte n'est qu'un dialogue entre les fées du récit et "leurs scurs fées" qui sont en nous. Ainsi du poète. II ne peut dire ou entendre un mot sans que celui-ci n'éveille en lui sa sceur-fèe, sans que ne s'établissent des connivences intimes entre le poète et le mot et, par le mot, entre le poète 
et le monde. Tout mol a donc son double en l'äme du poète, et ce mot-fée assure de nouvelle façon la solidite des liens entre le poète et la langue.

Saint-Denys Garneau franchit un dernier pas et ne craint plus d'affirmer, entre le mot et lui, une sorte d'identité, d'assimılation complète, puisque "le mot est lui-mème (le poète) en quelque sorte". Et de citer l'exemple du mot "oiseau", " cette part en lui-mème qui est oiseau". II est difficile d'alter plus loin dans cette direction: plus rien ne distingue le poète, ou une part de luiméme, de tel mot, de telle parole poétique, tant est grande la fusion substantielle entre eux. Dans cette identıfication, it n'y a plus de place pour l'angoisse d'ètre anéanti par l'autre pressenti comme un adversaire, ni pour l'affrontement de deux Je distincts, tels qu'on les découvrait dans les poèmes. Avouons toutefois qu'il semble difficile de tenir à la fois tous les types de relation qui surgissent dans la pensée de Saint-Denys Garneau. II s'est peutêtre laissé griser par la beauté des formules, une fols la paix retrouvée!

De tels liens entre le poète et le mot réveillent en lui l'instinct de possession: il " possède le mot", il est "libre du mot pour en jouer ". Le poète reprend donc l'initiative, apres avoir èté si longtemps la victime du verbe. II redevient le maitre du jeu et rappelle ses heureuses expériences espiégles du Jeu (O. AJE, 10) où ce faisant,

"Il vous arrange les mots comme si c'étaient de simples chansons" et "sous les mots il déplace toutes choses."

Plus tard, en mai 1938, dans un sursaut de sincérité, il reconnaîtra que " tout jeu n'est pas justifiable: on n'a pas le droit de jouer par les mots de ce qui ne comporte pas en nous de substance profonde" (O. J, 582). II était donc primordial, pour justifier la création poètique, que Saint-Denys Garneau se sente en relation de fraternité intime avec le mot et que toute distance soit abolie.

Un problème se pose : celui de l'importance qu'a revêtue jusqu'ici la relation subjective du poète avec la parole. N'y aurait-ll dans le mol pas d'autre substance que celle du poète lui-méme? Toute relation s'établirait-elle nécessairement en circuit fermé? Le poète ne ferait donc que se dire luimême, inlassablement. Quand on sait de quel "vide d'être", de quel néant et de quelle solitude il souffre, on peut se demander ce qu'il advient "du monde"! Les deux derniers poèmes étudiès semblaient en effet ignorer le monde et les rapports du poète avec le monde. Le Monologue apporte un correctıf qui paraît important.

En effet, dès que le mot apparaît comme être "vivant, substantiel", it manifeste les "possibilites de ses rapports au monde". il est plein, "lourd de sens". De plus, en jouant avec les mots, te poète rend " sensıble le jeu qu'il fait de toutes choses". Par le mot, le poète entre donc en communication, en communion avec le monde; mais c'est un univers encore marque par l'experience de l'homme. Toutefois, le "mot" ne présente qu'un aspect 
ponctuel des choses, "sans résonance". C'est la "Parole", c'est-à-dire la chaine des mots, "le deroulement sonore", le chant, qui met toutes choses en relation, car elle se présente comme un tout organisé et structuré dans son aspect linéaire. Si les choses restent disjointes dans l'isolement des mots, elles entrent en relation dans la Parole et plus encore dans le poème. Le tissage des liens se dèroule mystérieusement dans le cceur du poète, qui est le lieu, "le prisme" ou tous les rayons se croisent, fusionnent ou se différencient. Ainsi "la Parole brise la solitude de toutes choses" en les rapportant au cour du poète.

L'image du "prisme " permet à Saint-Denys Garneau de ramener à une certaine unité tant d'avenues ouvertes en des directions souvent opposées. Elle nous paraît cependant, si on la pousse jusqu'au bout, conduire à une conclusion contraire qui détruirait ce bel édifice. Le prisme n'est qu'un lieu de passage de la lumière, il agit comme catalyseur; il reste substantiellement différent des rayons qui le traversent, et étranger à ce qui se passe en lui. Que le poète soit le prisme, - Saint-Denys Garneau reprend la mẽme idée dans un autre passage du Journal (O.J. 487) sans se rendre compte probablement de l'ambiguitè de l'image - et voilà que s'elfondrent toutes les relations substantielles, de fraternité, d'identité, si laborieusement conquises! On aurait mauvaise grâce d'insister. Retenons seulement que le Monologue se présente comme un essai de synthèse de la pensée et de l'expérience de Saint-Denys Garneau sur le problème de la parole poétique.

Un poète ne fait jamais un exposé exhaustif sur la parole poétique, il livre nécessairement une part de son expérience personnelle dans l'acte de la création. Même si, dans les grandes lignes, on assiste toujours au même affrontement d'un homme avec le langage, cette expérience, au cours des mois et des années, ne peut être que multiforme et complexe, comme elle reste individuelle, originale et unique. Nous ne sommes donc pas surpris, en parcourant trois poèmes de Saint-Denys Garneau et un essai en prose concernant " la parole " ou "le mot ", d'avoir rencontré tant d'élèments divers, parfois contradicloires.

Le rapport de la parole aux choses qu'elle exprime ou qu'elle désigne, a posé peu de problemes. Pour Saint-Denys Garneau, la question n'est pas de savoir si les mots correspondent à la réalité sensible des choses concrètes du monde extérieur et l'expriment totalement. II n'a de souci que pour la réalité mystérieuse des choses telle qu'elle vit en lui. Ainsi le mot, la parole semblent ne jamais contenir ou signilier les choses dans leur réalité concrète, mais toujours dans leur rapport avec le poète, insérées dans son expérience humaine et spirituelle. Un jour Saint-Denys Garneau s'apercevra de ce danger. Remuè dans ses convictions par la lecture du livre d'Aimé Forest, Du consentement à l'être, il constate en lui-mème " un certain changement " dans son attitude et son rapport aux choses: il a peur d'avoir manque de respect 
envers la rèalitè des êtres, en cherchant en eux un " au-delà " (O.J. 540). Mais à celte date, il aura presque cessé d'écrire...

La relation de la parole au poète s'est révélée beaucoup plus complexe et incertaine. Outre les nombreuses oppositions ou contradictions qu'on a décelées au passage, deux aspects paraissent dominer cette relation. La passivité du poète d'abord est évidente. Malgré quelques affirmations, ici ou là, sur la liberté du poète de jouer des mots et, par eux, de l'univers, les trois poèmes étudiés montrent surtout le poète spectateur de ce qui se passe en lui. subissant l'action de la parole: le Monologue privilégie mème cette action par rapport à celle du poète qui, en dernière analyse, n'est que le prisme où tout s'opère. Un poéme a chantonné (O.PR, 166), plus tardif encore que l'essai en prose, insistera sur la longue et vaine attente du poete: malgré sa marche et sa quête tiévreuses, rien n'est venu à la lumière, rien n'a jailli, et le poète sombre dans une solitude et un silence plus tragiques. On dirait que le poème se joue presque sans lui, quand ce n'est pas contre lui.

L'on touche ainsi au second aspect de la relation. A peu près tout ce que Saint-Denys Garneau dit de la parole ou du poème, est une projection de son " arrière-conscience", une projection, sur la parole, de son mal intérieur. Tout se gâte au moment du passage de la parole de l'état intérieur á la forme articulée extérieure, donc au moment où le poète se livre. Rappelons-nous, en particulier, le drame vécu au moment de la parution du recueil Regards of jeux dans l'espace (O.J. 497): le poète avait surtout peur "d'être découvert *. Saint-Denys Garneau ne sortirait guère de lui-même, ce que confirmerait l'étude des thèmes de son œuvre: dès l'automne 1935, il porte moins d'intèrèt au monde des choses et des êtres qui l'entourent, pour se concentrer sur son propre problème, celui de son existence, de la recherche d'une issue à sa vie.

II nous parait enfin, en situant ce thème de la parole dans l'ensemble de "'ceuvre, que pour Saint-Denys Garneau la vision des choses et des êtres, leur captation par le regard et leur intime possession en lui étaient beaucoup plus importantes que leur expression dans l'écriture poétique. Le Journal, la Correspondance et maints poèmes trahissent cette préoccupation constante. Préoccupation qui apparait surtout dans Le Jeu, Spectacle de la danse (O.AJE, 12) et Le diable pour ma condamnation (O.PR, 186), ainsi que dans le Journal (O.J, 537) et la Correspondance: " ce que je cherche. c'est une sorte de possession du monde par l'esprit au moyen de l'art. Ce qu'on cherche au fond, c'est être toute chose, être l'absolu " (O.C, 941). L'ètat privilégié de la parole interieure en est une autre preuve. Etrange attitude pour un poete! Aússi finira-t-il par se taire, "réduit au silence", pour "consentir à l'être". La parole l'aurait-elle trompé? Nous dirions plutôt, comme nous le suggère Jean Le Moyne, que le poète, aux prises avec "la dissociation progressive de sa personne", aurait choisi de se concentrer sur l'essentiel et d'en finir avec la parole, devenue insupportable extériorisation de son ètre. 
1. Toutes nos références et les textes ètudiès renvoient a l'ouvrage Saint-Denys GARNEAU, OEuvres, edition critique par J. Brault \& B. Lacroix, Montréal. P.U.M., 1971. On utilisera les abreviatıons suivantes

O.C. : CEuvres, "Correspondance", et la page.

O.J. : CEuvres "Journal ".

O.Pr : CEuvres "Prose" (tels les nombreux articles préparés pour La Relève).

O.PR : Euvres "Poèmes retrouvés" (dont une partie a été publiée sous le titre "Les Solitudes".)

O.RJE: CEuvres "Regards et jeux dans l'espace".

2. Le poète devrail s'abstenir de deux sortes de conlidences, pense-t-il : celle des visions merveilleuses, des choses délıcates de l'âme. trésor qu'on ne répand pas sur la place publique: "l'âme et le cceur humain sont tellement délicals que parfois il me semble que toute parole qui les touche devrait être tue " (O.C. lettre à Yolande Leblanc, 21/8/1935. p. 963-64); celle des misères que la pudeur commanderatt de taire : ma misére, ma pauvreté, mon désert sont incommunicables" (O.J, 536).

3. A propos du poeme Silence et dans te Journal. Un manuscrit de Silence $(0$. Notes, p 1095) porte sur feuille volante, séparée du poème par un trait, la confidence suivante: " Parfois quand j'ecris (j'ai) l'impression que ces choses sont vraies. mais non pas immédiatement, qu'elles sont vraies ailleurs dans ma vie $n$. Cette confidence laisserait croire qu'opposé a m immédiatement ". notation plutōt temporelle, "ailleurs dans ma vie " signilierail : a un autre moment de ma vie, notation également temporelle. Tandis que le poeme semble assez clairement opposer "ailleurs" à " ici », dans un plan nettement spatial. Du reste, dans le même manuscrit, Saint-Denys Garneau, qui avait écrit d'abord le dernier vers ainsi :

"Et possède ailleurs dans l'éternel".

a bilfé les deux derniers mots comme s'il avait perçu que la notation d'éternité moditiait et peut-être affaiblissait çelle de l'Ailleurs. - D'autre part, comment ne pas être trappé par l'aspect proustien de cette confidence? - Dans le Journal, au cours de sa longue réflexion esthétique sur son art, il avoue avoir éprouvê dés son “ jeune áge, cette necessité de rejoindre la réalité. de la posseder ailleurs, plus parfaitement, eternellement o (c'est nous qui soulignons), (O.J. p. 442).

4. Thèse longuement développée dans sa réflexion esthétique de 1936, dans le Journal, (0.J. p. 430-444). J. Maritain, Frontiére de la poésio. p. 21, avait affirmé que e par la laçon dont il métamorphose l'univers passant dans son esprit, pour faire resplendır sur une matière une forme devinée dans les choses, l'artiste ... recompose, tel qu'en lui-méme enfin la poésie le change, un monde plus réel que ie réel offert aux sens" (c'est nous qui soulignons), el Anne Hébert. Poèmes. p. 68. le confirme: "cela devient une autre réalité, aussi vraie que la premiere $*$.

5. Ct. cette réllexion du poète dans Tous el chacun $(O, P R, 157)$ : au lieu de réaliser un beau marıge avec "tous et chacun réversibles". il n'a réussi qu'un accouplement. $\mathrm{Cl}$. aussi le Monologue $(0, \mathrm{Pr}, 289)$ : " on dit: "j'ai découvert un mot"... (ce) qui ne veut pas dire qu'on ait trouvé le nom de tel assemblage de matière ... mais qu'un mot nous est apparu tout à coup....

6. Ce vers: "Un tils..." a èté rajoute par l'auteur après la première facture du poème $(O$, Notes, p. 1096). De plus, ce grief est tout à fait logique avec la conclusion de Silence. il ne fallait pas parler'

7. Saint-Denys Garneau reproche aux mots les plus simples. comme le mot "arbre", d'être usès, "vides a force qu'on passe dessus". II faudrait trouver " un petit nombre de mots simples... saturés de l'étre des choses... trouver ces mots et les vivre. vivre toute leur plénitude. Avec cela faire un poème: ce poème serait vrai " (Lettres à ses amis. Montréal. HMH, 1967, Lettre à Robert Elie, sept. 
1936, p 227). Cl aussı J. Maritain, op. cif., p. 13. Plus explıcite encore est la rellexion du poete Rainer Maria Rılke dans "Les Cahiers de M.L. Brıgge". OEuvres, t.l, prose. Seuil, p. 544 : "Pour ecrire un seul vers, il faut avoir vu beaucoup de villes, d'hommes et de choses *, accumulé une trés tongue el très vaste experience de toute une vie: it faut avoir beaucoup de souvenirs, savoir les oublier et attendre qu'ils a deviennent en nous sang, regard, geste,... qu'ils ne se distinguent plus de nous". Alors seulement Il peut arriver que "se lève le premier mot d'un vers". 